\title{
Three-band tight-binding model for monolayers of group-VIB transition metal dichalcogenides
}

\author{
Gui-Bin Liu, ${ }^{1,2}$ Wen-Yu Shan, ${ }^{3}$ Yugui Yao, ${ }^{1}$ Wang Yao,,${ }^{2,}$ and Di Xiao ${ }^{3, \dagger}$ \\ ${ }^{1}$ School of Physics, Beijing Institute of Technology, Beijing 100081, China \\ ${ }^{2}$ Department of Physics and Center of Theoretical and Computational Physics, The University of Hong Kong, Hong Kong, China \\ ${ }^{3}$ Department of Physics, Carnegie Mellon University, Pittsburgh, Pennsylvania 15213, USA
}

(Received 28 May 2013; revised manuscript received 20 July 2013; published 26 August 2013)

\begin{abstract}
We present a three-band tight-binding (TB) model for describing the low-energy physics in monolayers of group-VIB transition metal dichalcogenides $M X_{2}(M=\mathrm{Mo}, \mathrm{W} ; X=\mathrm{S}, \mathrm{Se}, \mathrm{Te})$. As the conduction- and valence-band edges are predominantly contributed by the $d_{z^{2}}, d_{x y}$, and $d_{x^{2}-y^{2}}$ orbitals of $M$ atoms, the TB model is constructed using these three orbitals based on the symmetries of the monolayers. Parameters of the TB model are fitted from the first-principles energy bands for all $M X_{2}$ monolayers. The TB model involving only the nearest-neighbor $M-M$ hoppings is sufficient to capture the band-edge properties in the $\pm K$ valleys, including the energy dispersions as well as the Berry curvatures. The TB model involving up to the third-nearest-neighbor $M-M$ hoppings can well reproduce the energy bands in the entire Brillouin zone. Spin-orbit coupling in valence bands is well accounted for by including the on-site spin-orbit interactions of $M$ atoms. The conduction band also exhibits a small valley-dependent spin splitting which has an overall sign difference between $\operatorname{Mo} X_{2}$ and $\mathrm{W} X_{2}$. We discuss the origins of these corrections to the three-band model. The three-band TB model developed here is efficient to account for low-energy physics in $M X_{2}$ monolayers, and its simplicity can be particularly useful in the study of many-body physics and physics of edge states.
\end{abstract}

DOI: 10.1103/PhysRevB.88.085433

PACS number(s): 71.15.-m, 73.22.-f, 73.61.Le

\section{INTRODUCTION}

Recently, monolayers of group-VIB transition metal dichalcogenides $M X_{2}(M=\mathrm{Mo}, \mathrm{W} ; X=\mathrm{S}, \mathrm{Se})$ have attracted significant interest due to their extraordinary electronic and optical properties. These two-dimensional semiconductors possess a direct band gap ${ }^{1-5}$ in the visible frequency range and exhibit excellent mobility at room temperature, ${ }^{6-11}$ making them promising candidates for electronic and optoelectronic applications. ${ }^{12}$

$M X_{2}$ monolayers can be regarded as the semiconductor analog of graphene, with both the conduction- and valenceband edges located at the two corners of the first Brillouin zone (BZ), i.e., $K$ and $-K$ points [Fig. 1(c)]. Thus, electrons and holes acquire an extra valley degree of freedom, which may be used for information encoding and processing. ${ }^{13-17}$ Following earlier theoretical studies, ${ }^{13,14}$ it was predicted that inversion symmetry breaking in monolayer $M X_{2}$ gives rise to a valley-dependent optical transition selection rule, where interband transitions in $K$ and $-K$ valleys couple preferentially to left- and right-circularly polarized light. ${ }^{18,19}$ This prediction has led to the first experimental observations of dynamical pumping of valley polarization by circularly polarized light in monolayers of $\mathrm{MoS}_{2},{ }^{19-21}$ followed by the demonstration of electric control of valley circular dichroism in bilayer $\mathrm{MoS}_{2}{ }^{22}$ and valley coherence in monolayer $\mathrm{WSe}_{2} \cdot{ }^{23}$ Moreover, because of the giant spin-orbit coupling (SOC) in the material, ${ }^{24}$ the absence of inversion symmetry also allows a strong coupling between the spin and the valley degrees of freedom. ${ }^{18}$ These results suggest that monolayer $M X_{2}$ could possibly be the host for integrated spintronics and valleytronics.

In Ref. 18, where the valley-spin coupled physics is first predicted in monolayer $M X_{2}$, an effective two-band $\boldsymbol{k} \cdot \boldsymbol{p}$ model is given based on symmetry considerations, which suggests that the band-edge electrons and holes can be described as massive Dirac fermions. This $\boldsymbol{k} \cdot \boldsymbol{p}$ model has also been applied to study the transport, optical, and magnetic properties of $M X_{2}$ monolayers ${ }^{25-27}$ and bilayers. ${ }^{22,28}$ However, the $\boldsymbol{k} \cdot \boldsymbol{p}$ model is only valid close to the band edge. To obtain a more accurate description of the band structure, several tight-binding (TB) and $\boldsymbol{k} \cdot \boldsymbol{p}$ models have been recently introduced at the expense of including more orbitals into the Hamiltonian. ${ }^{29-32}$

In this paper, we develop a minimal symmetry-based three-band TB model using only the $M-d_{z^{2}}, d_{x y}$, and $d_{x^{2}-y^{2}}$ orbitals. We show that, by including only the nearest-neighbor (NN) hoppings, this TB model is sufficient to capture the band-edge properties in the $\pm K$ valleys, including the energy dispersions as well as the Berry curvatures. By including up to the third-nearest-neighbor (TNN) $M-M$ hoppings, our model can well reproduce the energy bands in the entire BZ. All parameters in our model are determined accurately by fitting the first-principles (FP) energy bands, and results for $X=\mathrm{Te}$ are also shown for systematical purpose although $M \mathrm{Te}_{2}$ monolayers are not realized experimentally now. SOC effects are studied under the approximation of on-site spin-orbit interaction, which results in a large valence-band spin splitting at the $K$ point. Besides, for the small but finite conduction-band spin splitting at $K$ recently noted, ${ }^{33-37}$ we reveal here a sign difference between $\operatorname{Mo} X_{2}$ and $\mathrm{W} X_{2}$, and show that such splitting can be partly accounted for by perturbative corrections to the three-band model. Our model provides a minimal starting point to include various interaction effects.

This paper is organized as follows. In Sec. II, we introduce our three-band TB model and fitting results. In Sec. III, SOC effects are studied. Conclusions are given in Sec. IV. In addition, an application of the TB model in zigzag nanoribbon is demonstrated in Appendix A. The relation between the $\boldsymbol{k} \cdot \boldsymbol{p}$ model in Ref. 18 and this TB model is shown in Appendix B. The FP method is given in Appendix C. 


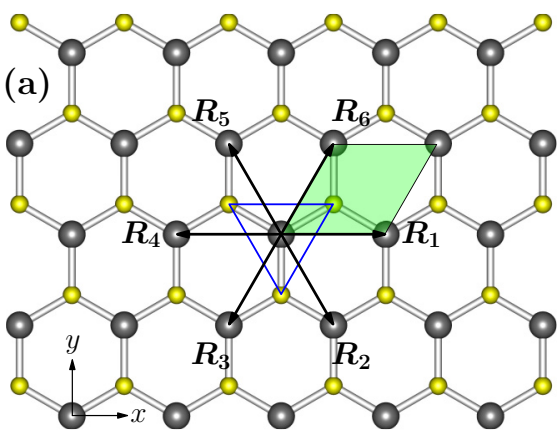

(b)

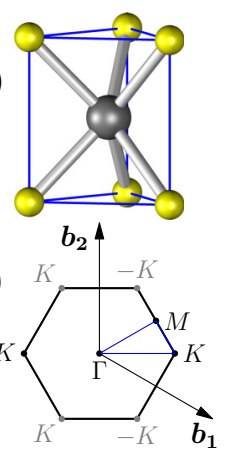

FIG. 1. (Color online) (a) Top view of monolayer $M X_{2}$. The large sphere is $M$ and the small sphere is $X$. $\boldsymbol{R}_{1}$ through $\boldsymbol{R}_{6}$ show the $M-M$ nearest neighbors. The shadowed diamond region shows the two-dimensional (2D) unit cell with lattice constant $a$. (b) Schematic for the structure of trigonal prismatic coordination, corresponding to the blue triangle in (a). (c) The 2D first Brillouin zone with special $\boldsymbol{k}$ points. $\boldsymbol{b}_{1}$ and $\boldsymbol{b}_{2}$ are the reciprocal basis vectors. The two inequivalent valleys $K$ and $-K$ are shown in black and their equivalent counterparts in gray.

\section{THE THREE-BAND TB MODEL}

For simplicity we first introduce the spinless model, and SOC is considered in the next section. In the following, we first analyze the symmetries and orbitals to determine the bases, then give the three-band TB model involving NN $M-M$ hoppings, and finally introduce up to TNN hoppings to improve the TB bands.

\section{A. Symmetries, orbitals, and bases}

Monolayer $M X_{2}$ has the $D_{3 h}$ point-group symmetry and its structure is shown in Fig. 1. From early theoretical studies ${ }^{38,39}$ and recent FP investigations ${ }^{24,34,40,41}$ we know that the Bloch states of monolayer $\mathrm{MoS}_{2}$ near the band edges mostly consist of Mo $d$ orbitals, especially the $d_{z^{2}}, d_{x y}$, and $d_{x^{2}-y^{2}}$ orbitals. Figure 2 clearly shows that contributions from $s$ orbitals are

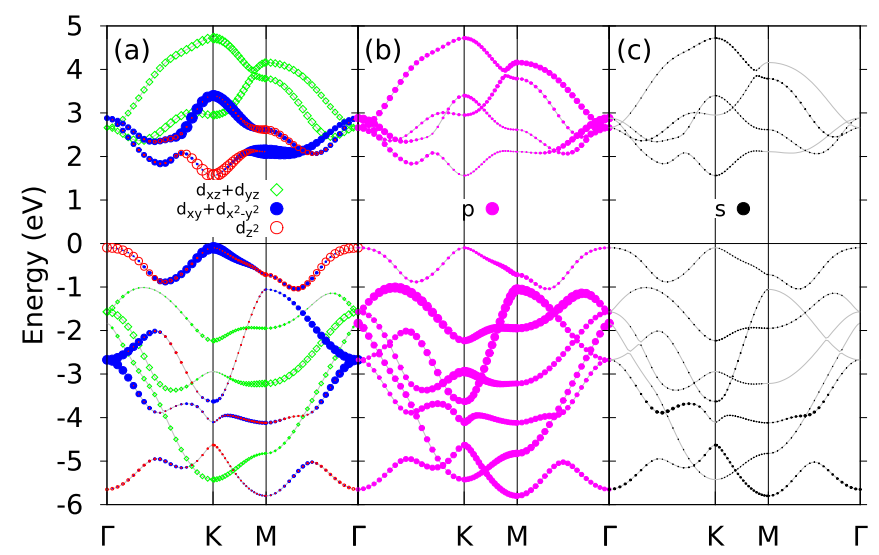

FIG. 2. (Color online) Orbital projected band structures for monolayer $\mathrm{MoS}_{2}$ from FP calculations. Fermi energy is set to zero. Symbol size is proportional to its population in corresponding state. (a) Contributions from Mo $d$ orbitals: blue dots for $d_{x y}$ and $d_{x^{2}-y^{2}}$, red open circles for $d_{z^{2}}$, and green open diamonds for $d_{x z}$ and $d_{y z}$. (b) Total $p$ orbitals, dominated by $\mathrm{S}$ atoms. (c) Total $s$ orbitals. negligible, those from $p$ orbitals are very small near the band edges, and $d_{z^{2}}, d_{x y}$, and $d_{x^{2}-y^{2}}$ orbitals are dominant components for conduction and valence bands. The trigonal prismatic coordination [Fig. 1(b)] splits the Mo $d$ orbitals into three categories: ${ }^{39} A_{1}^{\prime}\left\{d_{z^{2}}\right\}, E^{\prime}\left\{d_{x y}, d_{x^{2}-y^{2}}\right\}$, and $E^{\prime \prime}\left\{d_{x z}, d_{y z}\right\}$, where $A_{1}^{\prime}, E^{\prime}$, and $E^{\prime \prime}$ are the Mulliken notations for the irreducible representations (IRs) of point group $D_{3 h}$. The reflection symmetry by the $x-y$ plane, $\hat{\sigma}_{h}$, allows hybridization only between orbitals in $A_{1}^{\prime}$ and $E^{\prime}$ categories, leaving $E^{\prime \prime}$ decoupled from $A_{1}^{\prime}$ and $E^{\prime}$ bands [Fig. 2(a)]. In fact, the above analyses are also true for all monolayers of $M X_{2}$. Therefore, it is reasonable to construct a three-band TB model of monolayer $M X_{2}$ which can capture the main low-energy physics by considering $d-d$ hoppings using the minimal set of $M-d_{z^{2}}, d_{x y}$, and $d_{x^{2}-y^{2}}$ orbitals as bases. Obviously, using only the three $d$ orbitals and neglecting $X-p$ orbitals for the bases is an approximation, which is referred to as the "three-band approximation" hereinafter.

To conveniently describe the atomic bases by the symmetry of the $D_{3 h}$ point group, we denote them as $\left|\phi_{\mu}^{j}\right\rangle\left(\mu=1, \ldots, l_{j}\right)$ in terms of the $\mu$ th basis belonging to the $j$ th IR:

$$
\left|\phi_{1}^{1}\right\rangle=d_{z^{2}}, \quad\left|\phi_{1}^{2}\right\rangle=d_{x y}, \quad\left|\phi_{2}^{2}\right\rangle=d_{x^{2}-y^{2}},
$$

where $j=1$ stands for $A_{1}^{\prime}, j=2$ for $E^{\prime}$, and $l_{j}$ for the dimension of the $j$ th IR. Then the matrix elements of the Hamiltonian $\hat{H}$ can be easily obtained as $H_{\mu \mu^{\prime}}^{j j^{\prime}}(\boldsymbol{k})=\sum_{\boldsymbol{R}} e^{i \boldsymbol{k} \cdot \boldsymbol{R}} E_{\mu \mu^{\prime}}^{j j^{\prime}}(\boldsymbol{R})$ in which

$$
E_{\mu \mu^{\prime}}^{j j^{\prime}}(\boldsymbol{R})=\left\langle\phi_{\mu}^{j}(\boldsymbol{r})|\hat{H}| \phi_{\mu^{\prime}}^{j^{\prime}}(\boldsymbol{r}-\boldsymbol{R})\right\rangle
$$

is the hopping integral between the atomic orbitals $\left|\phi_{\mu}^{j}\right\rangle$ at $\mathbf{0}$ and $\left|\phi_{\mu^{\prime}}^{j^{\prime}}\right\rangle$ at lattice vector $\boldsymbol{R}$. Given $E_{\mu \mu^{\prime}}^{j j^{\prime}}(\boldsymbol{R})$, the hopping integrals to all neighboring sites can be generated by

$$
E^{j j^{\prime}}\left(\hat{g}_{n} \boldsymbol{R}\right)=D^{j}\left(\hat{g}_{n}\right) E^{j j^{\prime}}(\boldsymbol{R})\left[D^{j^{\prime}}\left(\hat{g}_{n}\right)\right]^{\dagger},
$$

where $D^{j}\left(\hat{g}_{n}\right)$ with dimension $l_{j} \times l_{j}$ is the matrix of the $j$ th IR and $E^{j j^{\prime}}(\boldsymbol{R})$ with dimension $l_{j} \times l_{j^{\prime}}$ is the matrix composed of $E_{\mu \mu^{\prime}}^{j j^{\prime}}(\boldsymbol{R}) . \hat{g}$ 's are a subset of the symmetry operations of $D_{3 h}$, $\left\{\hat{E}, \hat{C}_{3}, \hat{C}_{3}^{2}, \hat{\sigma}_{v}, \hat{\sigma}_{v}^{\prime}, \hat{\sigma}_{v}^{\prime \prime}\right\}$, where $\hat{E}$ is the identity operation, $\hat{C}_{3}$ is the rotation by $2 \pi / 3$ around the $z$ axis, $\hat{\sigma}_{v}$ is the reflection by the plane perpendicular to the $x-y$ plane and through the angular bisector of $\boldsymbol{R}_{1}$ and $\boldsymbol{R}_{6}$ in Fig. 1(a), and $\hat{\sigma}_{v}^{\prime}$ and $\hat{\sigma}_{v}^{\prime \prime}$ are obtained through rotating $\hat{\sigma}_{v}$ around the $z$ axis by $2 \pi / 3$ and $4 \pi / 3$, respectively. Using the above symmetry relation, we can reduce the parameters, i.e., the hopping integrals, to a minimal set. We emphasize that these symmetry-based $d-d$ hoppings include not only the direct $d-d$ interactions of $M$ atoms but also the indirect interactions mediated by $X$ - $p$ orbitals.

\section{B. Model with nearest-neighbor hoppings}

In this section, we introduce the three-band TB model involving only $\mathrm{NN} d-d$ hoppings, which is referred to as "NN TB" in the following. After determining each Hamiltonian matrix element, we get the three-band NN TB Hamiltonian 
TABLE I. Band energies at the high-symmetry $\boldsymbol{k}$ points analytically obtained from the TB Hamiltonian Eq. (4). The energies at each $\boldsymbol{k}$ point are in ascending order; $t_{12}>0$ is assumed.

\begin{tabular}{lcc}
\hline \hline$\Gamma=(0,0)$ & $K=\left(\frac{4 \pi}{3 a}, 0\right)$ & $M=\left(\frac{\pi}{a}, \frac{\pi}{\sqrt{3} a}\right)$ \\
\hline$\epsilon_{1}+6 t_{0}$ & $\epsilon_{2}-\frac{3}{2}\left(t_{11}+t_{22}\right)-3 \sqrt{3} t_{12}$ & $f_{1}-f_{2}{ }^{\mathrm{a}}$ \\
$\epsilon_{2}+3\left(t_{11}+t_{22}\right)$ & $\epsilon_{1}-3 t_{0}$ & $\epsilon_{2}+t_{11}-3 t_{22}$ \\
& $\epsilon_{2}-\frac{3}{2}\left(t_{11}+t_{22}\right)+3 \sqrt{3} t_{12}$ & $f_{1}+f_{2}$ \\
\hline \hline
\end{tabular}

${ }^{\mathrm{a}} f_{1}$ and $f_{2}$ are functions independent of $t_{1}$ :

$f_{1}=\frac{1}{2}\left(\epsilon_{1}+\epsilon_{2}\right)-t_{0}-\frac{3}{2} t_{11}+\frac{1}{2} t_{22}$,

$f_{2}=\frac{1}{2} \sqrt{\left(\epsilon_{1}-\epsilon_{2}-2 t_{0}+3 t_{11}-t_{22}\right)^{2}+64 t_{2}^{2}}$.

as

$$
H^{\mathrm{NN}}(\boldsymbol{k})=\left[\begin{array}{ccc}
h_{0} & h_{1} & h_{2} \\
h_{1}^{*} & h_{11} & h_{12} \\
h_{2}^{*} & h_{12}^{*} & h_{22}
\end{array}\right],
$$

in which

$$
\begin{gathered}
h_{0}=2 t_{0}(\cos 2 \alpha+2 \cos \alpha \cos \beta)+\epsilon_{1}, \\
h_{1}=-2 \sqrt{3} t_{2} \sin \alpha \sin \beta+2 i t_{1}(\sin 2 \alpha+\sin \alpha \cos \beta), \\
h_{2}=2 t_{2}(\cos 2 \alpha-\cos \alpha \cos \beta)+2 \sqrt{3} i t_{1} \cos \alpha \sin \beta, \\
h_{11}=2 t_{11} \cos 2 \alpha+\left(t_{11}+3 t_{22}\right) \cos \alpha \cos \beta+\epsilon_{2}, \\
h_{22}=2 t_{22} \cos 2 \alpha+\left(3 t_{11}+t_{22}\right) \cos \alpha \cos \beta+\epsilon_{2}, \\
h_{12}=\sqrt{3}\left(t_{22}-t_{11}\right) \sin \alpha \sin \beta \\
+4 i t_{12} \sin \alpha(\cos \alpha-\cos \beta), \\
(\alpha, \beta)=\left(\frac{1}{2} k_{x} a, \frac{\sqrt{3}}{2} k_{y} a\right), \\
t_{0}=E_{11}^{11}\left(\boldsymbol{R}_{1}\right), \quad t_{1}=E_{11}^{12}\left(\boldsymbol{R}_{1}\right), \quad t_{2}=E_{12}^{12}\left(\boldsymbol{R}_{1}\right), \\
t_{11}=E_{11}^{22}\left(\boldsymbol{R}_{1}\right), \quad t_{12}=E_{12}^{22}\left(\boldsymbol{R}_{1}\right), \quad t_{22}=E_{22}^{22}\left(\boldsymbol{R}_{1}\right),
\end{gathered}
$$

and $\epsilon_{j}$ is the on-site energy corresponding to the atomic orbital $\left|\phi_{\mu}^{j}\right\rangle$. Note that, for simplicity, we have assumed the orthogonality between each pair of different bases; therefore, the overlapping matrix of the bases is omitted and only the Hamiltonian matrix $H^{\mathrm{NN}}(\boldsymbol{k})$ is considered. Confined by the symmetry of the system, there are eight independent parameters in $H^{\mathrm{NN}}(\boldsymbol{k}): \epsilon_{1}, \epsilon_{2}, t_{0}, t_{1}, t_{2}, t_{11}, t_{12}$, and $t_{22}$.

To determine the eight parameters in the TB model accurately, we fit the band structures according to the FP results. There is no definitive strategy to fit the bands. In our case, since we are mostly interested in the low-energy physics near the $\pm K$ points and our analysis is entirely symmetry based, we fit the band energies at the high-symmetry $\boldsymbol{k}$ points, namely $\Gamma, K$, and $M$ (listed in Table I), together with least-squares fitting according to the energies of the conduction and valence bands near $K$.

By fitting the FP band structures of relaxed monolayers of $M X_{2}$ in both generalized-gradient approximation (GGA) and local-density approximation (LDA) cases, we get the TB parameters listed in Table II and the corresponding band structures shown in Fig. 3. The FP results (lattice parameters and band structures) obtained here are consistent with previous works. $^{24,41-45}$ In Fig. 3, by comparing the TB bands with the FP bands from $d_{z^{2}}, d_{x y}$, and $d_{x^{2}-y^{2}}$ orbitals, we can see that the former agree well with the latter near the conduction-band minimum (CBM) and valence-band maximum (VBM) at $K$ for all the $M X_{2}$ monolayers, but they significantly deviate from the latter in other regions. This is because the three-band approximation neglects the $p$ orbitals of $X$ atoms which still have substantial contributions to the conduction bands at $\Gamma$ and valence bands at $M$ [Fig. 2(b)]. Nevertheless, this simple NN TB model is sufficient to describe the physics of conduction and valence bands in $\pm K$ valleys. In addition, a trial model Hamiltonian of $M X_{2}$ zigzag nanoribbon based on this simple NN TB model can give reasonable edge states (see Appendix A).

We note that the band structure is very sensitive to the lattice constant: ${ }^{46-50}$ in Fig. 3(a) the valence-band energy at $\Gamma$ is higher than at $K$ by $4 \mathrm{meV}$, and in Figs. 3(i) and 3(j) the conduction-band energy at the dip near the midpoint of $\Gamma$ and $K$ is lower than at $K$ by 5 and $59 \mathrm{meV}$, respectively. This contradicts the observed direct band gaps. This is related to the different relaxed lattice constants between GGA and LDA (GGA tends to overestimate the lattice constant, whereas LDA underestimates it; see Table II). This, however, has little effect on our fitting at the $K$ point.

\section{Model with up to third-nearest-neighbor hoppings}

To reproduce the energy bands in the entire BZ, we further consider up to TNN $M-M$ hoppings. By the same symmetry-based procedure, we derive the three-band TNN model Hamiltonian $H^{\mathrm{TNN}}(\boldsymbol{k})$ as

$$
H^{\mathrm{TNN}}(\boldsymbol{k})=\left[\begin{array}{ccc}
V_{0} & V_{1} & V_{2} \\
V_{1}^{*} & V_{11} & V_{12} \\
V_{2}^{*} & V_{12}^{*} & V_{22}
\end{array}\right],
$$

in which

$$
\begin{aligned}
V_{0}= & \epsilon_{1}+2 t_{0}(2 \cos \alpha \cos \beta+\cos 2 \alpha) \\
& +2 r_{0}(2 \cos 3 \alpha \cos \beta+\cos 2 \beta) \\
& +2 u_{0}(2 \cos 2 \alpha \cos 2 \beta+\cos 4 \alpha),
\end{aligned}
$$

$$
\begin{aligned}
\operatorname{Re}\left[V_{1}\right]= & -2 \sqrt{3} t_{2} \sin \alpha \sin \beta+2\left(r_{1}+r_{2}\right) \sin 3 \alpha \sin \beta \\
& -2 \sqrt{3} u_{2} \sin 2 \alpha \sin 2 \beta,
\end{aligned}
$$

$\operatorname{Im}\left[V_{1}\right]=2 t_{1} \sin \alpha(2 \cos \alpha+\cos \beta)+2\left(r_{1}-r_{2}\right) \sin 3 \alpha \cos \beta$

$$
+2 u_{1} \sin 2 \alpha(2 \cos 2 \alpha+\cos 2 \beta) \text {, }
$$

$$
\begin{aligned}
\operatorname{Re}\left[V_{2}\right]= & +2 t_{2}(\cos 2 \alpha-\cos \alpha \cos \beta) \\
& -\frac{2}{\sqrt{3}}\left(r_{1}+r_{2}\right)(\cos 3 \alpha \cos \beta-\cos 2 \beta) \\
& +2 u_{2}(\cos 4 \alpha-\cos 2 \alpha \cos 2 \beta),
\end{aligned}
$$

$$
\begin{aligned}
\operatorname{Im}\left[V_{2}\right]= & 2 \sqrt{3} t_{1} \cos \alpha \sin \beta \\
& +\frac{2}{\sqrt{3}} \sin \beta\left(r_{1}-r_{2}\right)(\cos 3 \alpha+2 \cos \beta) \\
& +2 \sqrt{3} u_{1} \cos 2 \alpha \sin 2 \beta,
\end{aligned}
$$


TABLE II. Fitted parameters of the three-band NN TB model based on the FP band structures of monolayer $M X_{2}$ using both GGA and LDA. $a$ and $z_{X-X}$ are the relaxed lattice constant and $X-X$ distance in the $z$ direction, respectively. The energy parameters $\epsilon_{1}$ through $t_{22}$ are in units of $\mathrm{eV}$.

\begin{tabular}{|c|c|c|c|c|c|c|c|c|c|c|}
\hline & $a(\AA)$ & $z_{X-X}(\AA)$ & $\epsilon_{1}$ & $\epsilon_{2}$ & $t_{0}$ & $t_{1}$ & $t_{2}$ & $t_{11}$ & $t_{12}$ & $t_{22}$ \\
\hline & \multicolumn{10}{|c|}{ GGA } \\
\hline $\mathrm{MoS}_{2}$ & 3.190 & 3.130 & 1.046 & 2.104 & -0.184 & 0.401 & 0.507 & 0.218 & 0.338 & 0.057 \\
\hline $\mathrm{WS}_{2}$ & 3.191 & 3.144 & 1.130 & 2.275 & -0.206 & 0.567 & 0.536 & 0.286 & 0.384 & -0.061 \\
\hline $\mathrm{MoSe}_{2}$ & 3.326 & 3.345 & 0.919 & 2.065 & -0.188 & 0.317 & 0.456 & 0.211 & 0.290 & 0.130 \\
\hline $\mathrm{WSe}_{2}$ & 3.325 & 3.363 & 0.943 & 2.179 & -0.207 & 0.457 & 0.486 & 0.263 & 0.329 & 0.034 \\
\hline $\mathrm{MoTe}_{2}$ & 3.557 & 3.620 & 0.605 & 1.972 & -0.169 & 0.228 & 0.390 & 0.207 & 0.239 & 0.252 \\
\hline \multirow[t]{2}{*}{$\mathrm{WTe}_{2}$} & 3.560 & 3.632 & 0.606 & 2.102 & -0.175 & 0.342 & 0.410 & 0.233 & 0.270 & 0.190 \\
\hline & \multicolumn{10}{|c|}{ LDA } \\
\hline $\mathrm{MoS}_{2}$ & 3.129 & 3.115 & 1.238 & 2.366 & -0.218 & 0.444 & 0.533 & 0.250 & 0.360 & 0.047 \\
\hline $\mathrm{WS}_{2}$ & 3.132 & 3.126 & 1.355 & 2.569 & -0.238 & 0.626 & 0.557 & 0.324 & 0.405 & -0.076 \\
\hline $\mathrm{MoSe}_{2}$ & 3.254 & 3.322 & 1.001 & 2.239 & -0.222 & 0.350 & 0.488 & 0.244 & 0.314 & 0.129 \\
\hline $\mathrm{WSe}_{2}$ & 3.253 & 3.338 & 1.124 & 2.447 & -0.242 & 0.506 & 0.514 & 0.305 & 0.353 & 0.025 \\
\hline $\mathrm{MoTe}_{2}$ & 3.472 & 3.598 & 0.618 & 2.126 & -0.202 & 0.254 & 0.423 & 0.241 & 0.263 & 0.269 \\
\hline $\mathrm{WTe}_{2}$ & 3.476 & 3.611 & 0.623 & 2.251 & -0.209 & 0.388 & 0.442 & 0.272 & 0.295 & 0.200 \\
\hline
\end{tabular}

$$
\begin{aligned}
V_{11}= & \epsilon_{2}+\left(t_{11}+3 t_{22}\right) \cos \alpha \cos \beta+2 t_{11} \cos 2 \alpha \\
& +4 r_{11} \cos 3 \alpha \cos \beta+2\left(r_{11}+\sqrt{3} r_{12}\right) \cos 2 \beta \\
& +\left(u_{11}+3 u_{22}\right) \cos 2 \alpha \cos 2 \beta+2 u_{11} \cos 4 \alpha,
\end{aligned}
$$

$$
\begin{aligned}
\operatorname{Re}\left[V_{12}\right]= & \sqrt{3}\left(t_{22}-t_{11}\right) \sin \alpha \sin \beta+4 r_{12} \sin 3 \alpha \sin \beta \\
& +\sqrt{3}\left(u_{22}-u_{11}\right) \sin 2 \alpha \sin 2 \beta,
\end{aligned}
$$

$$
\begin{aligned}
\operatorname{Im}\left[V_{12}\right]= & 4 t_{12} \sin \alpha(\cos \alpha-\cos \beta) \\
& +4 u_{12} \sin 2 \alpha(\cos 2 \alpha-\cos 2 \beta),
\end{aligned}
$$

and

$$
\begin{aligned}
V_{22}= & \epsilon_{2}+\left(3 t_{11}+t_{22}\right) \cos \alpha \cos \beta+2 t_{22} \cos 2 \alpha \\
& +2 r_{11}(2 \cos 3 \alpha \cos \beta+\cos 2 \beta) \\
& +\frac{2}{\sqrt{3}} r_{12}(4 \cos 3 \alpha \cos \beta-\cos 2 \beta) \\
& +\left(3 u_{11}+u_{22}\right) \cos 2 \alpha \cos 2 \beta+2 u_{22} \cos 4 \alpha .
\end{aligned}
$$

The additional parameters are defined as

$$
\begin{aligned}
r_{0} & =E_{11}^{11}\left(\tilde{\boldsymbol{R}}_{1}\right), \quad r_{1}=E_{11}^{12}\left(\tilde{\boldsymbol{R}}_{1}\right), \quad r_{2}=E_{12}^{12}\left(\tilde{\boldsymbol{R}}_{1}\right), \\
r_{11} & =E_{11}^{22}\left(\tilde{\boldsymbol{R}}_{1}\right), \quad r_{12}=E_{12}^{22}\left(\tilde{\boldsymbol{R}}_{1}\right),
\end{aligned}
$$

and

$u_{0}=E_{11}^{11}\left(2 \boldsymbol{R}_{1}\right), \quad u_{1}=E_{11}^{12}\left(2 \boldsymbol{R}_{1}\right), \quad u_{2}=E_{12}^{12}\left(2 \boldsymbol{R}_{1}\right)$,

$u_{11}=E_{11}^{22}\left(2 \boldsymbol{R}_{1}\right), \quad u_{12}=E_{12}^{22}\left(2 \boldsymbol{R}_{1}\right), \quad u_{22}=E_{22}^{22}\left(2 \boldsymbol{R}_{1}\right)$,

in which $\tilde{\boldsymbol{R}}_{1}=\boldsymbol{R}_{1}+\boldsymbol{R}_{2}$ is one of the next-NN vectors and $2 \boldsymbol{R}_{1}$ is one of the TNN vectors.

The fitted parameters for $H^{\mathrm{TNN}}(\boldsymbol{k})$ are listed in Table III and the corresponding bands are shown in Fig. 4 from which we can see that the three TB bands agree well with the FP ones contributed by $d_{z^{2}}, d_{x y}$, and $d_{x^{2}-y^{2}}$ orbitals in the entire BZ. The well-reproduced bands mean that effective masses can be obtained accurately by this TNN TB model. In addition, we show the Berry curvatures calculated using this TB model in Fig. 5(b) which shows good agreement with the result in
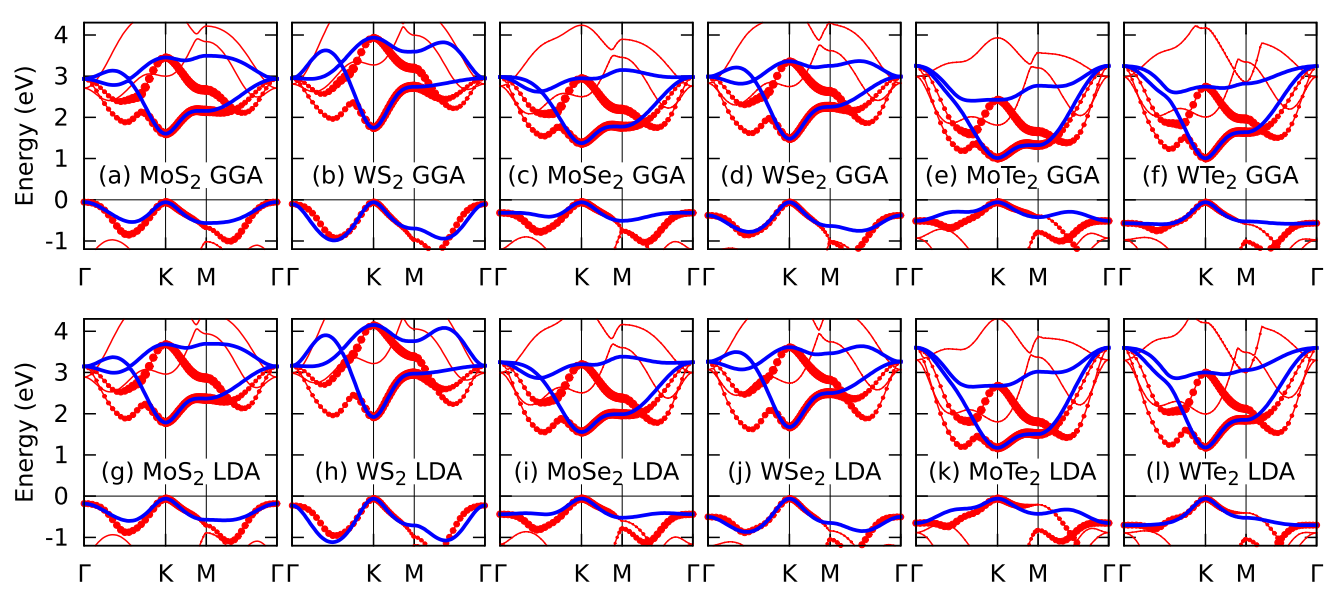

FIG. 3. (Color online) The NN TB band structures (blue or dark curves) of $M X_{2}$ monolayers compared with the FP ones (red or gray curves and dots). VBMs are shifted to zero. The dots show the band components from $d_{z^{2}}, d_{x y}$, and $d_{x^{2}-y^{2}}$ orbitals, with which the TB bands should compare. (a-f) GGA and (g-1) LDA. 
TABLE III. Fitted parameters (in units of eV) for the three-band TNN TB model based on the FP bands in both GGA and LDA cases.

\begin{tabular}{|c|c|c|c|c|c|c|c|c|c|c|}
\hline & $\begin{array}{l}\epsilon_{1} \\
r_{2}\end{array}$ & $\begin{array}{c}\epsilon_{2} \\
r_{11}\end{array}$ & $\begin{array}{c}t_{0} \\
r_{12}\end{array}$ & $\begin{array}{l}t_{1} \\
u_{0}\end{array}$ & $\begin{array}{l}t_{2} \\
u_{1}\end{array}$ & $\begin{array}{l}t_{11} \\
u_{2}\end{array}$ & $\begin{array}{l}t_{12} \\
u_{11}\end{array}$ & $\begin{array}{l}t_{22} \\
u_{12}\end{array}$ & $\begin{array}{c}r_{0} \\
u_{22}\end{array}$ & $r_{1}$ \\
\hline & & & & & GGA & & & & & \\
\hline \multirow[t]{2}{*}{$\mathrm{MoS}_{2}$} & 0.683 & 1.707 & -0.146 & -0.114 & 0.506 & 0.085 & 0.162 & 0.073 & 0.060 & -0.236 \\
\hline & 0.067 & 0.016 & 0.087 & -0.038 & 0.046 & 0.001 & 0.266 & -0.176 & -0.150 & \\
\hline \multirow[t]{2}{*}{$\mathrm{WS}_{2}$} & 0.717 & 1.916 & -0.152 & -0.097 & 0.590 & 0.047 & 0.178 & 0.016 & 0.069 & -0.261 \\
\hline & 0.107 & -0.003 & 0.109 & -0.054 & 0.045 & 0.002 & 0.325 & -0.206 & -0.163 & \\
\hline \multirow[t]{2}{*}{$\mathrm{MoSe}_{2}$} & 0.684 & 1.546 & -0.146 & -0.130 & 0.432 & 0.144 & 0.117 & 0.075 & 0.039 & -0.209 \\
\hline & 0.069 & 0.052 & 0.060 & -0.042 & 0.036 & 0.008 & 0.272 & -0.172 & -0.150 & \\
\hline \multirow[t]{2}{*}{$\mathrm{WSe}_{2}$} & 0.728 & 1.655 & -0.146 & -0.124 & 0.507 & 0.117 & 0.127 & 0.015 & 0.036 & -0.234 \\
\hline & 0.107 & 0.044 & 0.075 & -0.061 & 0.032 & 0.007 & 0.329 & -0.202 & -0.164 & \\
\hline \multirow[t]{2}{*}{$\mathrm{MoTe}_{2}$} & 0.588 & 1.303 & -0.226 & -0.234 & 0.036 & 0.400 & 0.098 & 0.017 & 0.003 & -0.025 \\
\hline & -0.169 & 0.082 & 0.051 & 0.057 & 0.103 & 0.187 & -0.045 & -0.141 & 0.087 & \\
\hline \multirow[t]{2}{*}{$\mathrm{WTe}_{2}$} & 0.697 & 1.380 & -0.109 & -0.164 & 0.368 & 0.204 & 0.093 & 0.038 & -0.015 & -0.209 \\
\hline & 0.107 & 0.115 & 0.009 & -0.066 & $\begin{array}{c}0.011 \\
\text { LDA }\end{array}$ & -0.013 & 0.312 & -0.177 & -0.132 & \\
\hline \multirow[t]{2}{*}{$\mathrm{MoS}_{2}$} & 0.820 & 1.931 & -0.176 & -0.101 & 0.531 & 0.084 & 0.169 & 0.070 & 0.070 & -0.252 \\
\hline & 0.084 & 0.019 & 0.093 & -0.043 & 0.047 & 0.005 & 0.304 & -0.192 & -0.162 & \\
\hline \multirow[t]{2}{*}{$\mathrm{WS}_{2}$} & 0.905 & 2.167 & -0.175 & -0.090 & 0.611 & 0.043 & 0.181 & 0.008 & 0.075 & -0.282 \\
\hline & 0.127 & 0.001 & 0.114 & -0.063 & 0.047 & 0.004 & 0.374 & -0.224 & -0.177 & \\
\hline \multirow[t]{2}{*}{$\mathrm{MoSe}_{2}$} & 0.715 & 1.687 & -0.154 & -0.134 & 0.437 & 0.124 & 0.119 & 0.072 & 0.048 & -0.248 \\
\hline & 0.090 & 0.066 & 0.045 & -0.067 & 0.041 & 0.005 & 0.327 & -0.194 & -0.151 & \\
\hline \multirow[t]{2}{*}{$\mathrm{WSe}_{2}$} & 0.860 & 1.892 & -0.152 & -0.125 & 0.508 & 0.094 & 0.129 & 0.009 & 0.044 & -0.278 \\
\hline & 0.129 & 0.059 & 0.058 & -0.090 & 0.039 & 0.001 & 0.392 & -0.224 & -0.165 & \\
\hline \multirow[t]{2}{*}{$\mathrm{MoTe}_{2}$} & 0.574 & 1.410 & -0.148 & -0.173 & 0.333 & 0.203 & 0.186 & 0.127 & 0.007 & -0.280 \\
\hline & 0.067 & 0.073 & 0.081 & -0.054 & 0.008 & 0.037 & 0.145 & -0.078 & 0.035 & \\
\hline \multirow[t]{2}{*}{$\mathrm{WTe}_{2}$} & 0.675 & 1.489 & -0.124 & -0.159 & 0.362 & 0.196 & 0.101 & 0.044 & -0.009 & -0.250 \\
\hline & 0.129 & 0.131 & -0.007 & -0.086 & 0.012 & -0.020 & 0.361 & -0.193 & -0.129 & \\
\hline
\end{tabular}

Ref. 51. We note that around the $\Gamma$ point the conduction bands with the lowest energies are made of $d_{x z}, d_{y z}$, and $X$-p orbitals, which cannot be captured by our three-band model.
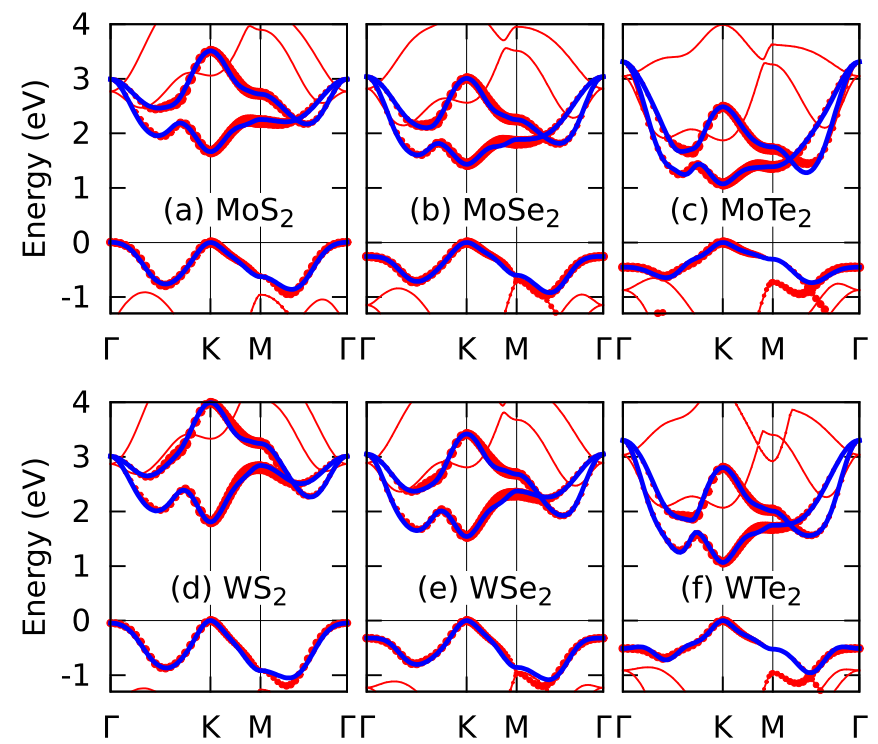

FIG. 4. (Color online) Energy bands from the TNN TB model (blue or dark curves) of $M X_{2}$ monolayers compared with the FP ones in the GGA case (red or gray curves and dots). The dots show the band components from $d_{z^{2}}, d_{x y}$, and $d_{x^{2}-y^{2}}$ orbitals, with which the TB bands should compare.
It should be noted that energy bands are only one aspect of physical properties and hence not enough to capture all physics. We also calculated the $\boldsymbol{k}$-resolved degree of circular polarization for absorbed photons, $\eta(\boldsymbol{k})$. As shown in Ref. 19, $\eta(\boldsymbol{k})$ has the same sign in each region of $1 / 6$ of the BZ around each $K$ or $-K$ point and exhibits a high degree of polarization in most of each region. We can see that the $\eta(\boldsymbol{k})$ calculated using the TB model here can give correct values in the large neighborhood of $\pm K$, but not in the small region around $\Gamma$ [see Figs. 5(a) and 5(c)] due to the limitation of the three-band approximation. It can be seen from Figs. 3 and 5 that the three-band approximation works well around the $\pm K$ valleys
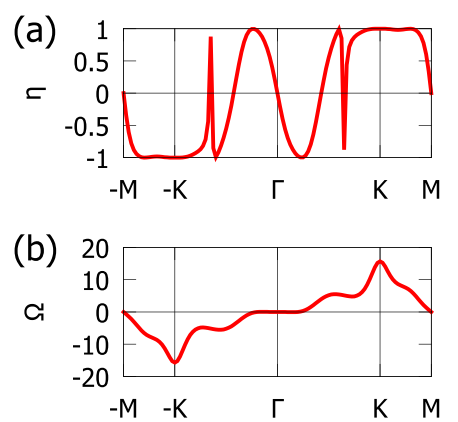

(c)

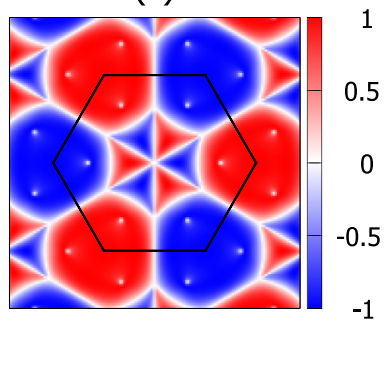

FIG. 5. (Color online) Quantities from the TNN TB for monolayer $\mathrm{MoS}_{2}$ under GGA parameters: (a) degree of circular polarization, $\eta(\boldsymbol{k})$, and (b) Berry curvature $\Omega(\boldsymbol{k})$ in units of $\AA^{2}$ along $\boldsymbol{k}$ path $-M \rightarrow-K \rightarrow \Gamma \rightarrow K \rightarrow M$. (c) Color map of $\eta(\boldsymbol{k})$, where the hexagon shows the BZ. 
TABLE IV. The SOC splitting of the valence band at $K \Delta_{\text {SOC }}^{v}$, the second-order corrected SOC parameter $\lambda$, the SOC splitting of the conduction band at $K$ from the second-order perturbation theory $\Delta_{\mathrm{SOC}}^{\mathrm{c}(\mathrm{pt})}$ and from FP bands $\Delta_{\mathrm{SOC}}^{\mathrm{c}(\mathrm{FP})}$ (GGA case), and the energy parameters in Eqs. (30) and (31). $E_{1,2}=E_{+1}-E_{+2}, E_{-1,0}=E_{-1}-E_{0}$, and $E_{1,0}=E_{+1}-E_{0}$. All quantities are in units of eV.

\begin{tabular}{lcccrrrr}
\hline \hline & $\Delta_{\text {SOC }}^{\mathrm{v}}$ & $\lambda$ & $\Delta_{\text {SOC }}^{\mathrm{c}(\mathrm{pt})}$ & \multicolumn{1}{c}{$\Delta_{\text {SOC }}^{\mathrm{c}(\mathrm{FP})}$} & $E_{1,2}$ & $E_{-1,0}$ & $E_{1,0}$ \\
\hline $\mathrm{MoS}_{2}$ & 0.148 & 0.073 & 0.003 & -0.003 & 4.840 & 1.395 & 3.176 \\
$\mathrm{WS}_{2}$ & 0.430 & 0.211 & 0.026 & 0.029 & 5.473 & 1.526 & 3.667 \\
$\mathrm{MoSe}_{2}$ & 0.184 & 0.091 & 0.007 & -0.021 & 4.296 & 1.128 & 2.862 \\
$\mathrm{WSe}_{2}$ & 0.466 & 0.228 & 0.038 & 0.036 & 4.815 & 1.267 & 3.275 \\
$\mathrm{MoTe}_{2}$ & 0.215 & 0.107 & 0.015 & -0.034 & 3.991 & 0.798 & 2.918 \\
$\mathrm{WTe}_{2}$ & 0.486 & 0.237 & 0.059 & 0.051 & 4.412 & 1.004 & 3.347 \\
\hline \hline
\end{tabular}

and also the valence-band $\Gamma$ point, where $d$ orbitals dominate, but not in the $k$-space region where $X$ - $p$ orbitals are important.

\section{SPIN-ORBIT COUPLING}

\section{A. The model with spin-orbit coupling}

Due to the heavy transition-metal $M$ atom, its SOC can be large. The large SOC of monolayer $M X_{2}$ is a fascinating feature which leads to its rich physics. For simplicity, here we approximate the SOC by considering only the on-site contribution, namely, the $\boldsymbol{L} \cdot \boldsymbol{S}$ term from $M$ atoms. Using the bases $\left\{\left|d_{z^{2}}, \uparrow\right\rangle,\left|d_{x y}, \uparrow\right\rangle,\left|d_{x^{2}-y^{2}}, \uparrow\right\rangle,\left|d_{z^{2}}, \downarrow\right\rangle,\left|d_{x y}, \downarrow\right\rangle,\left|d_{x^{2}-y^{2}}, \downarrow\right\rangle\right\}$, we get the SOC contribution to the Hamiltonian as

$$
H^{\prime}=\lambda \boldsymbol{L} \cdot \boldsymbol{S}=\frac{\lambda}{2}\left[\begin{array}{cc}
L_{z} & 0 \\
0 & -L_{z}
\end{array}\right],
$$

in which

$$
L_{z}=\left[\begin{array}{ccc}
0 & 0 & 0 \\
0 & 0 & 2 i \\
0 & -2 i & 0
\end{array}\right]
$$

is the matrix of $\hat{L}_{z}$ ( $z$ component of the orbital angular momentum) in bases of $d_{z^{2}}, d_{x y}$, and $d_{x^{2}-y^{2}}$, and $\lambda$ characterizes the strength of the SOC. Note that, under the three bases, the matrix elements of $\hat{L}_{x}$ and $\hat{L}_{y}$ are all zeros. Then we get the full TB Hamiltonian with SOC as follows:

$$
\begin{aligned}
H_{\text {SOC }}(\boldsymbol{k}) & =I_{2} \otimes H_{0}(\boldsymbol{k})+H^{\prime} \\
& =\left[\begin{array}{cc}
H_{0}(\boldsymbol{k})+\frac{\lambda}{2} L_{z} & 0 \\
0 & H_{0}(\boldsymbol{k})-\frac{\lambda}{2} L_{z}
\end{array}\right],
\end{aligned}
$$

in which $I_{2}$ is the $2 \times 2$ identity matrix and $H_{0}=H^{\mathrm{NN}}$ or $H^{\mathrm{TNN}}$. The above Hamiltonian is block diagonal, which means that the spin $z$ component is not mixed by the SOC and hence is still a good quantum number due to the $\hat{\sigma}_{h}$ symmetry. From Eq. (27) we can easily know that at the $K$ point the SOC interaction splits VBM by $\Delta_{\text {SOC }}^{\mathrm{v}}=2 \lambda$ and leaves CBM still degenerate (see detailed discussions in Sec. III B). The valence-band SOC (or spin) splittings are listed in the first column of Table IV. The bands from both the NN and the TNN TB Hamiltonians with SOC are shown in Fig. 6 for $\mathrm{Mo} X_{2}$. It can be seen that the NN TB bands agree well with the FP ones only for the conduction and valence bands near the $K$ point, while the TNN TB bands agree well in the entire BZ.
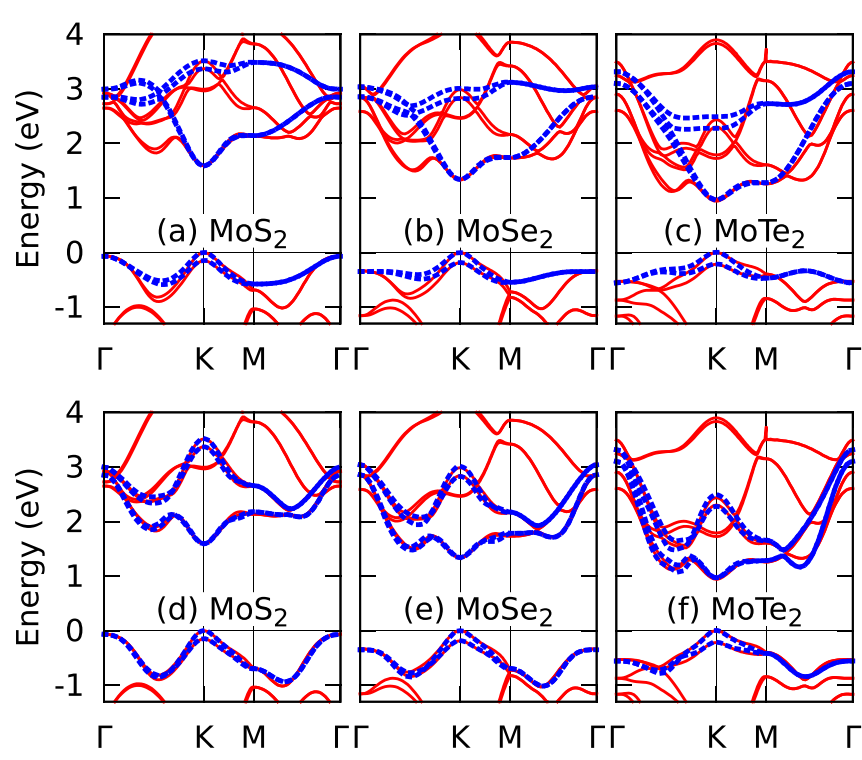

FIG. 6. (Color online) Energy bands of monolayers $\mathrm{Mo} X_{2}$ with SOC. Thick blue dashed curves are the TB bands: $(\mathrm{a}-\mathrm{c})$ from the NN TB model and (d-f) from the TNN TB model. Thin red solid curves are FP results with GGA. VBMs are shifted to zero.

Although the NN TB model is not as accurate as the TNN one, it can still give reasonable results for low-energy physics. Taking monolayer $\mathrm{MoS}_{2}$, for example, to test the NN TB model with SOC, we calculated the valence-band SOC splittings and the Berry curvatures and the spin Berry curvatures, shown in Fig. 7. The valley contrasting SOC splittings $E_{\mathrm{v} \uparrow}(\boldsymbol{k})-E_{\mathrm{v} \downarrow}(\boldsymbol{k})$ between the two spin split-off valence bands is clearly shown in Fig. 7(a), which agrees well with the result in Ref. 24. The Berry curvatures ${ }^{52,53}$ and spin Berry curvatures ${ }^{54}$ are all peaked at $\pm K$ points, and the former have opposite signs between $K$ and $-K$ [Fig. 7(b)] while the latter have the same signs between $K$ and $-K$ [Fig. 7(c)]. These lead to the valley Hall effect and the spin Hall effect when an in-plane electric field exists. ${ }^{18}$ The TB results shown in Figs. 7(b) and 7(c) agree quite well with the FP results in Ref. 51. Therefore, the NN TB model is sufficient to describe correctly the physics in $\pm K$ valleys.

\section{B. The SOC splitting of the conduction band}

To first order of the SOC strength, the TB model for monolayer $M X_{2}$ here can only reproduce the large spin splitting of the valence band at $K$, i.e., $\Delta_{\text {SOC }}^{\mathrm{v}}$, but gives no spin splitting of the conduction band at $K$, denoted by $\Delta_{\text {SOC }}^{\mathrm{c}}$. In fact, the conduction-band spin splitting (CBSS) is not zero but a finite small value ${ }^{33-37}$ and has been analyzed for $\mathrm{MoS}_{2}$ by previous works. ${ }^{31,55}$ Similar to the strong valley-spin coupling in the valence band, ${ }^{18}$ the CBSS is also valley dependent due to the time-reversal symmetry and leads to weak valley-spin coupling. Through a careful examination of the FP results, we note here that the CBSSs of $\operatorname{Mo} X_{2}$ have opposite signs to those of $\mathrm{W} X_{2}$, if $\Delta_{\mathrm{SOC}}^{\mathrm{c}}$ is defined as the energy difference $E_{\mathrm{c} \uparrow}-E_{\mathrm{c} \downarrow}$ at the $K$ point (see Table IV and Fig. 8). By analyzing the FP data, we know that CBSS is induced by small contributions from $M-d_{x z}, d_{y z}$, and $X-p_{x}, p_{y}$ orbitals. Here we go beyond the three-band approximation and show that a second-order 


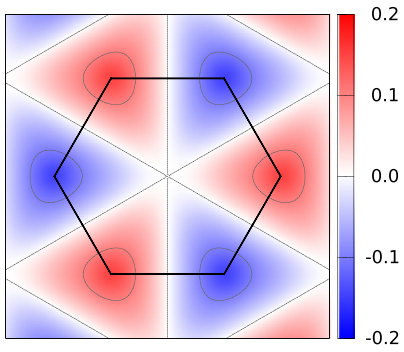

(a)

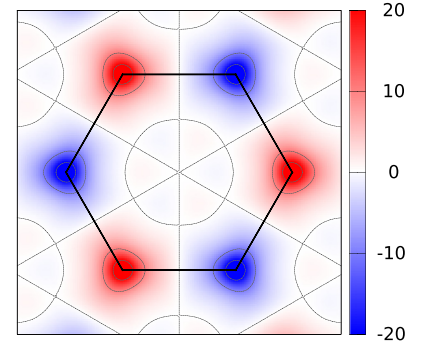

(b)

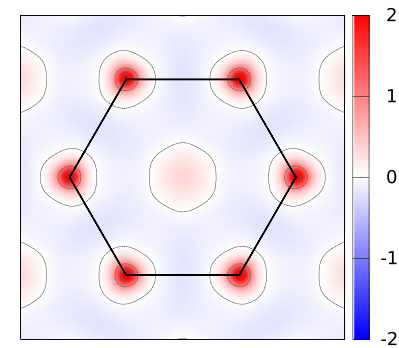

(c)

FIG. 7. (Color online) Contour maps in the $\boldsymbol{k}$ space for monolayer $\mathrm{MoS}_{2}$ from the NN TB model (using the GGA parameters): (a) the valence-band SOC splittings in units of eV, (b) the Berry curvatures, and (c) the spin Berry curvatures in units of $\AA^{2}$. The hexagons show the BZ. The gray thin curves are the contour lines corresponding to their tick values on the color bars.

perturbation correction involving $M-d_{x z}$ and $d_{y z}$ orbitals can partly explain the CBSSs.

FP wave functions show that, at the $K$ point, the Bloch waves, one below and four above the band gap, are dominantly composed of $d_{+2}, d_{0}, d_{-1}, d_{-2}$, and $d_{+1}$ orbitals in ascending order of energies, respectively, in the case without SOC, ${ }^{56}$ in which $d_{ \pm 2}=\frac{1}{\sqrt{2}}\left(d_{x^{2}-y^{2}} \pm i d_{x y}\right), d_{0}=d_{z^{2}}$, and $d_{ \pm 1}=$ $\frac{1}{\sqrt{2}}\left(d_{x z} \pm i d_{y z}\right)$. Accordingly, we assume that the five Bloch states are completely composed of the aforementioned five $d$ orbitals, respectively, which is a good approximation shown by the following results. To incorporate the contributions to CBSS from $d_{ \pm 1}$ (i.e., $d_{x z}$ and $d_{y z}$ ) orbitals, we make a second-order perturbation for the SOC interaction $H^{\prime}=\lambda \boldsymbol{L} \cdot \boldsymbol{S}$ through the Löwdin partitioning equation: ${ }^{57,58}$

$$
H_{m m^{\prime}}^{(2)}=\frac{1}{2} \sum_{l} H_{m l}^{\prime} H_{l m^{\prime}}^{\prime}\left[\frac{1}{E_{m}-E_{l}}+\frac{1}{E_{m^{\prime}}-E_{l}}\right],
$$

in which $H_{m l}^{\prime}=\left\langle d_{m}\left|H^{\prime}\right| d_{l}\right\rangle(m= \pm 2,0$ and $l= \pm 1)$ and $E_{m}$ is the band energy at $K$ corresponding to the $d_{m}$ orbital. Thus, the contributions from $d_{ \pm 1}$ orbitals are folded into an effective second-order SOC interaction in bases $\left\{d_{+2}, d_{0}, d_{-2}\right\} \otimes\{\uparrow, \downarrow\}$ as follows:

$$
H^{\prime(2)}=\operatorname{diag}\left\{0, \frac{-\lambda^{2}}{E_{+1}-E_{+2}}, \frac{-3 \lambda^{2}}{2\left(E_{+1}-E_{0}\right)}, \frac{-3 \lambda^{2}}{2\left(E_{-1}-E_{0}\right)}, \frac{\lambda^{2}}{E_{-2}-E_{-1}}, 0\right\} \text {. }
$$

Considering the first-order SOC interaction under the same bases, $H^{\prime(1)}=\operatorname{diag}\{\lambda,-\lambda, 0,0,-\lambda, \lambda\}$, finally we get the second-order corrected splittings

$$
\begin{gathered}
\Delta_{\text {SOC }}^{\mathrm{v}}=2 \lambda+\frac{\lambda^{2}}{E_{+1}-E_{+2}}, \\
\Delta_{\text {SOC }}^{\mathrm{c}}=\frac{3}{2} \lambda^{2}\left[\frac{1}{\left(E_{-1}-E_{0}\right)}-\frac{1}{\left(E_{+1}-E_{0}\right)}\right] .
\end{gathered}
$$

We first get the second-order corrected $\lambda$ by solving Eq. (30) and then put it into Eq. (31) to get $\Delta_{\text {SOC }}^{\mathrm{c}}$. The obtained

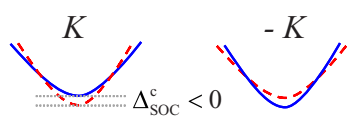

$\operatorname{Mo} X_{2}$

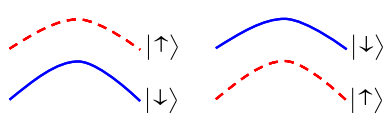

FIG. 8. (Color online) Schematic for the conduction- and valenceband spin splittings in the $\pm K$ valleys for $\mathrm{MoX}_{2}$ (left) and $\mathrm{WX}_{2}$ (right). Red dashed curves are spin-up states and blue solid ones spin-down states. The conduction-band spin splitting has an overall sign change between $\mathrm{MoX}_{2}$ and $\mathrm{W} X_{2}$. Crossings exist for the spinsplit conduction bands of $\mathrm{Mo} X_{2}$.
CBSSs from perturbation, $\Delta_{\mathrm{SOC}}^{\mathrm{c}(\mathrm{pt})}$, are listed in Table IV and compared with the FP results, $\Delta_{\mathrm{SOC}}^{\mathrm{c}(\mathrm{FP})}$. The signed CBSS avoids the spurious coincidence $\Delta_{\text {SOC }}^{\mathrm{c}(\mathrm{pt})}=\left|\Delta_{\text {SOC }}^{\mathrm{c}(\mathrm{FP})}\right|=3 \mathrm{meV}$ for $\mathrm{MoS}_{2}$ (see Table IV). We can see that the CBSSs determined by Eq. (31) agree very well with the FP splittings for $\mathrm{WX}_{2}$, but not for $\mathrm{MoX}_{2}$. We attribute these to the competition of the two origins of CBSS: (i) the second-order perturbation due to the coupling to the remote $d_{x z}$ and $d_{y z}$ orbitals and (ii) the first-order effect from the small component of $X-p_{x}$ and $p_{y}$ orbitals. Equation (31) contains only the origin (i) but not (ii). The $\mathrm{W}$ atom is heavier than the Mo atom; therefore, the W- $d$ orbitals are the dominant contribution of the CBSSs over $X$ - $p$ orbitals and thus Eq. (31) works well for $\mathrm{WX}_{2}$, whereas for $\mathrm{MoX}_{2}, X-p$ orbitals become non-negligible for CBSSs relative to Mo- $d$ orbitals and Eq. (31) breaks down for $\mathrm{Mo} X_{2}$. More rigorous treatments involving $X-p$ orbitals are needed for correctly describing the CBSSs of $\mathrm{Mo} X_{2}$, which is out of the scope of this paper.

We also note that band crossings exist for the spin-split conduction bands of $\mathrm{MoX} \mathrm{X}_{2}$, but not for $\mathrm{WX}_{2}$, as demonstrated in Fig. 8. The distance between the crossing and the $K$ point increases from $\mathrm{MoS}_{2}(\sim 0.052 \pi / a)$ to $\operatorname{MoSe}_{2}(\sim 0.152 \pi / a)$, and to $\operatorname{MoTe}_{2}(\sim 0.222 \pi / a)$. The band crossing arises from the spin dependence in the effective mass. At the $K$ point of $M X_{2}$, the spin-down carrier has larger band gap and thus heavier 
effective mass (flatter band) than the spin-up one. ${ }^{18}$ Combining the different sign of CBSS, the bands shift differently for $\mathrm{Mo} X_{2}$ and $\mathrm{W} X_{2}$ resulting in the crossings in $\mathrm{Mo} X_{2}$ but not in $\mathrm{W} X_{2}$. In addition, for different $\operatorname{Mo} X_{2}$, larger CBSS leads to larger distance of the crossing from the $K$ points. Because of the trigonal warping, the distances along the $K-\Gamma$ and $K-M$ directions have small difference, which is not shown in Fig. 8, and crossing appears in the $K-M$ but not the $K-\Gamma$ direction for $\mathrm{MoTe}_{2}$ due to its relatively large CBSS.

\section{CONCLUSIONS}

In this paper, we have developed a minimal symmetry-based three-band TB model for monolayers of $M X_{2}$ using only the $M-d_{z^{2}}, d_{x y}$, and $d_{x^{2}-y^{2}}$ orbitals. When only NN $M-M$ hoppings are included, this TB model is sufficient to capture the band-edge properties in the $\pm K$ valleys, including the energy dispersions as well as the Berry curvatures. By including up to the TNN $M-M$ hoppings, the model can well reproduce the energy bands in the entire BZ. In spite of the simple NN TB model, it can describe reasonably the edge states of zigzag $M X_{2}$ ribbon that consist of $d_{z^{2}}, d_{x y}$, and $d_{x^{2}-y^{2}}$ orbitals. SOC is introduced through the approximation of on-site $\boldsymbol{L} \cdot \boldsymbol{S}$ interactions in the heavy $M$ atoms, which lead to the giant SOC splittings of the valence bands at $K$. In addition, we analyzed the relatively small CBSSs at $K$ through a second-order perturbation involving $d_{x z}$ and $d_{y z}$ orbitals, which works quite well for $\mathrm{WX}_{2}$ but not for $\mathrm{MoX} X_{2}$. This is attributed to the $X$ - $p$ orbitals not presented in our model. We also pointed out that the signed CBSSs have different signs between $\mathrm{W} X_{2}$ and
Mo $X_{2}$. The three-band TB model developed here is efficient to account for low-energy physics in $M X_{2}$ monolayers, and its simplicity can be particularly useful in the study of many-body physics and the physics of edge states.

\section{ACKNOWLEDGMENTS}

The work was supported by the HKSAR Research Grant Council with Grant No. HKU706412P and the Croucher Foundation under the Croucher Innovation Award (G.B.L. and W.Y.); the National Basic Research Program of China 973 Program with Grant No. 2013CB934500 and the Basic Research Funds of Beijing Institute of Technology with Grant No. 20121842003 (G.B.L.); the National Basic Research Program of China 973 Program with Grant No. 2011CBA00100, the National Natural Science Foundation of China with Grants No. 11225418 and No. 11174337, and the Specialized Research Fund for the Doctoral Program of Higher Education of China with Grant No. 20121101110046 (Y.Y.); and the U.S. Department of Energy, Office of Basic Energy Sciences, Materials Sciences and Engineering Division (W.Y.S. and D.X.).

\section{APPENDIX A: MODEL FOR ZIGZAG NANORIBBON}

In this Appendix, we apply the three-band NN TB model to study $M X_{2}$ nanoribbons. Taking a zigzag nanoribbon in the $x$ direction with width $W$, for example, there are $W$ formula units in the $y$ direction, namely $M X_{2} \times W$, within an $x$-direction unit translational cell. The matrix elements of the Hamiltonian have three types:

$$
\begin{gathered}
H_{n \gamma, n \gamma^{\prime}}^{\text {ribbon }}=\delta_{\gamma \gamma^{\prime}} e_{\gamma}+e^{i \boldsymbol{k} \cdot \boldsymbol{R}_{1}} E_{\gamma \gamma^{\prime}}\left(\boldsymbol{R}_{1}\right)+e^{i \boldsymbol{k} \cdot \boldsymbol{R}_{4}} E_{\gamma \gamma^{\prime}}\left(\boldsymbol{R}_{4}\right) \quad(n=1, \ldots, W), \\
H_{n \gamma,(n-1) \gamma^{\prime}}^{\mathrm{ribbon}}=e^{i \boldsymbol{k} \cdot \boldsymbol{R}_{2}} E_{\gamma \gamma^{\prime}}\left(\boldsymbol{R}_{2}\right)+e^{i \boldsymbol{k} \cdot \boldsymbol{R}_{3}} E_{\gamma \gamma^{\prime}}\left(\boldsymbol{R}_{3}\right) \quad(n=2, \ldots, W), \\
H_{n \gamma,(n+1) \gamma^{\prime}}^{\mathrm{ribbon}}=e^{i \boldsymbol{k} \cdot \boldsymbol{R}_{5}} E_{\gamma \gamma^{\prime}}\left(\boldsymbol{R}_{5}\right)+e^{i \boldsymbol{k} \cdot \boldsymbol{R}_{6}} E_{\gamma \gamma^{\prime}}\left(\boldsymbol{R}_{6}\right) \quad(n=1, \ldots, W-1),
\end{gathered}
$$

in which $\gamma, \gamma^{\prime} \in\left\{\begin{array}{l}1 \\ 1\end{array}, \frac{2}{1}, 2\right\}, e_{1}^{1}=\epsilon_{1}$, and $e_{1}^{2}=e_{2}^{2}=\epsilon_{2}$. Then we can obtain the $3 W \times 3 W$ Hamiltonian matrix for the zigzag nanoribbon as follows:

$$
H^{\text {ribbon }}\left(k_{x}\right)=\left[\begin{array}{ccccc}
h_{1}^{\prime} & h_{2}^{\prime \dagger} & & & \\
h_{2}^{\prime} & h_{1}^{\prime} & h_{2}^{\prime \dagger} & & \\
& h_{2}^{\prime} & h_{1}^{\prime} & \ddots & \\
& & \ddots & \ddots & h_{2}^{\prime \dagger} \\
& & & h_{2}^{\prime} & h_{1}^{\prime}
\end{array}\right],
$$

in which $h_{1}^{\prime} \equiv H_{n n}^{\text {ribbon }}, h_{2}^{\prime} \equiv H_{n, n-1}^{\text {ribbon }}$, and

$$
\begin{gathered}
h_{1}^{\prime}=\left[\begin{array}{ccc}
\epsilon_{1}+2 \cos \left(k_{x} a\right) t_{0} & 2 i \sin \left(k_{x} a\right) t_{1} & 2 \cos \left(k_{x} a\right) t_{2} \\
-2 i \sin \left(k_{x} a\right) t_{1} & \epsilon_{2}+2 \cos \left(k_{x} a\right) t_{11} & 2 i \sin \left(k_{x} a\right) t_{12} \\
2 \cos \left(k_{x} a\right) t_{2} & -2 i \sin \left(k_{x} a\right) t_{12} & \epsilon_{2}+2 \cos \left(k_{x} a\right) t_{22}
\end{array}\right], \\
h_{2}^{\prime}=\left[\begin{array}{ccc}
2 \cos \left(\frac{1}{2} k_{x} a\right) t_{0} & i \sin \left(\frac{1}{2} k_{x} a\right)\left(t_{1}-\sqrt{3} t_{2}\right) & -\frac{1}{2} \cos \left(\frac{1}{2} k_{x} a\right)\left(\sqrt{3} t_{1}+t_{2}\right) \\
-i \sin \left(\frac{1}{2} k_{x} a\right)\left(t_{1}+\sqrt{3} t_{2}\right) & \frac{1}{2} \cos \left(\frac{1}{2} k_{x} a\right)\left(t_{11}+3 t_{22}\right) & -i \sin \left(\frac{1}{2} k_{x} a\right)\left(\frac{\sqrt{3}}{2} t_{11}+2 t_{12}-\frac{\sqrt{3}}{2} t_{22}\right) \\
\cos \left(\frac{1}{2} k_{x} a\right)\left(\sqrt{3} t_{1}-t_{2}\right) & -i \sin \left(\frac{1}{2} k_{x} a\right)\left(\frac{\sqrt{3}}{2} t_{11}-2 t_{12}-\frac{\sqrt{3}}{2} t_{22}\right) & \frac{1}{2} \cos \left(\frac{1}{2} k_{x} a\right)\left(3 t_{11}+t_{22}\right)
\end{array}\right] .
\end{gathered}
$$




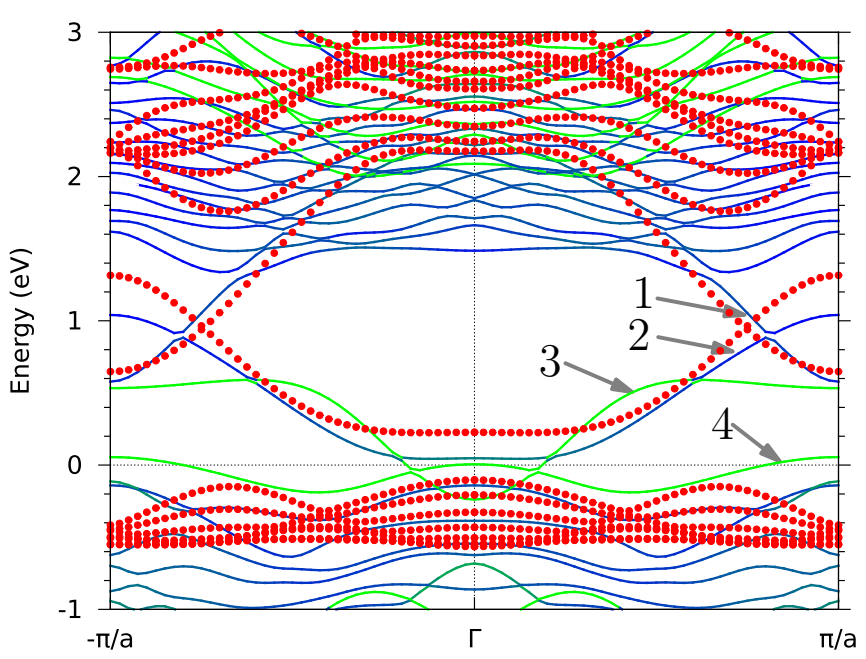

FIG. 9. (Color online) The energy bands for zigzag $\mathrm{MoS}_{2}$ nanoribbon with width $W=8$. Red dots are bands from the TB model using the GGA parameters. Curves are the FP bands, in which blue shows the contributions from the $d_{z^{2}}, d_{x y}$, and $d_{x^{2}-y^{2}}$ orbitals and green for other orbitals. For the bands labeled 1 through 4 , see the text.

The energy bands of a zigzag $\mathrm{MoS}_{2}$ nanoribbon with $W=8$ (using the GGA parameters in Table II) from both the TB model and FP calculations are given in Fig. 9. From the FP results, we know that bands 1 and 2 shown by arrows in Fig. 9 are the edge states from the $d_{z^{2}}, d_{x y}$, and $d_{x^{2}-y^{2}}$ orbitals of Mo atoms at the two edges of the ribbon; band 3 is from the Mo- $d_{y z}$ orbital at the Mo-terminated edge; and band 4 is from the $\mathrm{S}-p_{y}$ and $p_{z}$ orbitals at the S-terminated edge. Due to the neglect of $d_{x z}, d_{y z}$, and S- $p$ orbitals in the TB model, band 3 and 4 do not exist in the TB bands. Nevertheless, bands 1 and 2 are given by the TB model reasonably. Therefore, the simple NN TB model for $M X_{2}$ zigzag ribbon can give satisfactory results, if the edge state bands 1 and 2 are the focus of a study.

\section{APPENDIX B: THE TWO-BAND $k \cdot p$ MODEL}

It is clear from Fig. 3 that the three-band NN TB model is sufficient to describe the physics of conduction and valence bands in the $K$ valley (also true for the $-K$ valley due to the time-reversal symmetry). Thus, we can expand Eq. (4) in the $\pm K$ valleys to any order required and then reduce it to a twoband $\boldsymbol{k} \cdot \boldsymbol{p}$ model in the Löwdin partitioning method. ${ }^{57,59,60}$ Using $\left|\psi_{\mathrm{c}}^{\tau}\right\rangle=\left|d_{z^{2}}\right\rangle$ and $\left|\psi_{\mathrm{v}}^{\tau}\right\rangle=\frac{1}{\sqrt{2}}\left(\left|d_{x^{2}-y^{2}}\right\rangle+i \tau\left|d_{x y}\right\rangle\right)(\tau=$ \pm is the valley index) as bases, the obtained two-band $\boldsymbol{k} \cdot \boldsymbol{p}$ model up to third order in $\boldsymbol{k}$ (relative to $\tau K$ ) is

$$
\begin{aligned}
H_{k p}^{(1)}(\boldsymbol{k} ; \tau) & =\left[\begin{array}{cc}
\Delta / 2 & a t\left(\tau k_{x}-i k_{y}\right) \\
a t\left(\tau k_{x}+i k_{y}\right) & -\Delta / 2
\end{array}\right], \\
H_{k p}^{(2)}(\boldsymbol{k} ; \tau)= & H_{k p}^{(1)}(\boldsymbol{k} ; \tau) \\
& +a^{2}\left[\begin{array}{cc}
\gamma_{1} k^{2} & \gamma_{3}\left(\tau k_{x}+i k_{y}\right)^{2} \\
\gamma_{3}\left(\tau k_{x}-i k_{y}\right)^{2} & \gamma_{2} k^{2}
\end{array}\right],
\end{aligned}
$$

$$
\begin{aligned}
H_{k p}^{(3)}(\boldsymbol{k} ; \tau)= & H_{k p}^{(2)}(\boldsymbol{k} ; \tau) \\
& +a^{3}\left[\begin{array}{ll}
\gamma_{4} \tau k_{x}\left(k_{x}^{2}-3 k_{y}^{2}\right) & \gamma_{6} k^{2}\left(\tau k_{x}-i k_{y}\right) \\
\gamma_{6} k^{2}\left(\tau k_{x}+i k_{y}\right) & \gamma_{5} \tau k_{x}\left(k_{x}^{2}-3 k_{y}^{2}\right)
\end{array}\right],
\end{aligned}
$$

in which $\Delta$ is the band gap at $K, t$ and $\gamma_{1}-\gamma_{6}$ are energy parameters, and $k^{2}=k_{x}^{2}+k_{y}^{2}$. Equation (B1) is the massive Dirac Hamiltonian given in Ref. 18 which was derived just this way, and Eqs. (B2) and (B3) are consistent with previous works. ${ }^{29,31}$ In Fig. 10, the bands of monolayer $\mathrm{MoS}_{2}$ from $H_{k p}^{(1)}$ capture the main physics in the valley but neglect the details such as the anisotropic dispersion (the trigonal warping) and the electron-hole asymmetry, the bands from $H_{k p}^{(2)}$ recover the aforementioned missing details, and the bands from $H_{k p}^{(3)}$ agree with the FP bands perfectly.

When SOC is considered to first order, Eq. (27) is still valid and we can get

$$
H_{k p s o}^{(n)}(\boldsymbol{k} ; \tau, s)=H_{k p}^{(n)}(\boldsymbol{k} ; \tau)+\left[\begin{array}{cc}
0 & 0 \\
0 & \tau s \lambda
\end{array}\right],
$$

where $s= \pm 1$ is the spin index ( +1 for $\uparrow$ and -1 for $\downarrow)$ since spin is a good quantum number. The $\tau s \lambda$ term in Eq. (B4) appears in the form of the product of the valley index $\tau$, the spin index $s$, and the SOC parameter $\lambda$, which implies the rich physics due to the SOC-induced coupling of valley and spin described in Ref. 18.

\section{APPENDIX C: FP BAND STRUCTURE CALCULATIONS}

The FP band structures used for fitting the parameters were calculated by the VASP package $e^{61,62}$ using the projectoraugmented wave method. ${ }^{63,64}$ Exchange-correlation functionals of both GGA ${ }^{65}$ and LDA ${ }^{66,67}$ were used to give comparable results. The energy cutoff of the plane-wave basis was set to $400 \mathrm{eV}$ and the convergence criterion was $10^{-6} \mathrm{eV}$. A $\Gamma$ centered $\boldsymbol{k}$ mesh of $10 \times 10 \times 1$ was used and layer separation was greater than $15 \AA$. For all monolayers of $M X_{2}$, lattice constants were optimized and atomic positions were relaxed until the force on each atom was less than $0.005 \mathrm{eV} / \AA$.
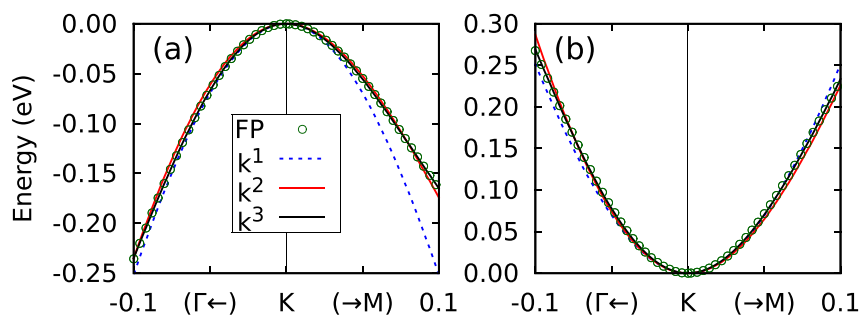

FIG. 10. (Color online) (a) Valence and (b) conduction bands in the $K$ valley of monolayer $\mathrm{MoS}_{2}$, within the range of $0.1 \times \frac{2 \pi}{a}$ in $\Gamma$ and $M$ directions. Open circles are FP results (GGA case). Blue dashed, red solid, and black solid curves are the bands from the two-band $\boldsymbol{k} \cdot \boldsymbol{p}$ model of $H_{k p}^{(1)}, H_{k p}^{(2)}$, and $H_{k p}^{(3)}$, respectively. CBMs and VBMs are both shifted to 0. $a=3.190 \AA$ and $\Delta=1.663 \mathrm{eV}$ for all. Other fitted parameters are the following: $t=1.105 \mathrm{eV}$ for $H_{k p}^{(1)} ; t=1.059 \mathrm{eV}$, $\gamma_{1}=0.055 \mathrm{eV}, \gamma_{2}=0.077 \mathrm{eV}$, and $\gamma_{3}=-0.123 \mathrm{eV}$ for $H_{k p}^{(2)} ; t=$ $1.003 \mathrm{eV}, \gamma_{1}=0.196 \mathrm{eV}, \gamma_{2}=-0.065 \mathrm{eV}, \gamma_{3}=-0.248 \mathrm{eV}, \gamma_{4}=$ $0.163 \mathrm{eV}, \gamma_{5}=-0.094 \mathrm{eV}$, and $\gamma_{6}=-0.232 \mathrm{eV}$ for $H_{k p}^{(3)}$. 
*wangyao@hku.hk

†dixiao@cmu.edu

${ }^{1}$ K. F. Mak, C. Lee, J. Hone, J. Shan, and T. F. Heinz, Phys. Rev. Lett. 105, 136805 (2010).

${ }^{2}$ A. Splendiani, L. Sun, Y. Zhang, T. Li, J. Kim, C.-Y. Chim, G. Galli, and F. Wang, Nano Lett. 10, 1271 (2010).

${ }^{3}$ S. Tongay, J. Zhou, C. Ataca, K. Lo, T. S. Matthews, J. Li, J. C. Grossman, and J. Wu, Nano Lett. 12, 5576 (2012).

${ }^{4}$ J. S. Ross, S. Wu, H. Yu, N. Ghimire, A. Jones, G. Aivazian, J. Yan, D. Mandrus, D. Xiao, W. Yao, and X. Xu, Nat. Commun. 4, 1474 (2013).

${ }^{5}$ H. Zeng, G.-B. Liu, J. Dai, Y. Yan, B. Zhu, R. He, L. Xie, S. Xu, X. Chen, W. Yao, and X. Cui, Sci. Rep. 3, 1608 (2013).

${ }^{6}$ B. Radisavljevic, A. Radenovic, J. Brivio, V. Giacometti, and A. Kis, Nat. Nanotechnol. 6, 147 (2011).

${ }^{7}$ D. Lembke and A. Kis, ACS Nano 6, 10070 (2012).

${ }^{8}$ M.-W. Lin, L. Liu, Q. Lan, X. Tan, K. S. Dhindsa, P. Zeng, V. M. Naik, M. M.-C. Cheng, and Z. Zhou, J. Phys. D 45, 345102 (2012).

${ }^{9}$ W. Bao, X. Cai, D. Kim, K. Sridhara, and M. S. Fuhrer, Appl. Phys. Lett. 102, 042104 (2013).

${ }^{10}$ S. Larentis, B. Fallahazad, and E. Tutuc, Appl. Phys. Lett. 101, 223104 (2012).

${ }^{11}$ H. Fang, S. Chuang, T. C. Chang, K. Takei, T. Takahashi, and A. Javey, Nano Lett. 12, 3788 (2012).

${ }^{12}$ Q. H. Wang, K. Kalantar-Zadeh, A. Kis, J. N. Coleman, and M. S. Strano, Nat. Nanotechnol. 7, 699 (2012).

${ }^{13}$ D. Xiao, W. Yao, and Q. Niu, Phys. Rev. Lett. 99, 236809 (2007).

${ }^{14}$ W. Yao, D. Xiao, and Q. Niu, Phys. Rev. B 77, 235406 (2008).

${ }^{15}$ O. Gunawan, Y. P. Shkolnikov, K. Vakili, T. Gokmen, E. P. De Poortere, and M. Shayegan, Phys. Rev. Lett. 97, 186404 (2006).

${ }^{16}$ A. Rycerz, J. Tworzydlo, and C. W. J. Beenakker, Nat. Phys. 3, 172 (2007).

${ }^{17}$ Z. Zhu, A. Collaudin, B. F. W. Kang, and K. Behnia, Nat. Phys. 8, 89 (2012).

${ }^{18}$ D. Xiao, G.-B. Liu, W. Feng, X. Xu, and W. Yao, Phys. Rev. Lett. 108, 196802 (2012).

${ }^{19}$ T. Cao, G. Wang, W. Han, H. Ye, C. Zhu, J. Shi, Q. Niu, P. Tan, E. Wang, B. Liu, and J. Feng, Nat. Commun. 3, 887 (2012).

${ }^{20}$ K. F. Mak, K. He, J. Shan, and T. F. Heinz, Nat. Nanotechnol. 7, 494 (2012).

${ }^{21}$ H. Zeng, J. Dai, W. Yao, D. Xiao, and X. Cui, Nat. Nanotechnol. 7, 490 (2012).

${ }^{22}$ S. Wu, J. S. Ross, G.-B. Liu, G. Aivazian, A. Jones, Z. Fei, W. Zhu, D. Xiao, W. Yao, D. Cobden, and X. Xu, Nat. Phys. 9, 149 (2013).

${ }^{23}$ A. M. Jones, H. Yu, N. J. Ghimire, S. Wu, G. Aivazian, J. S. Ross, B. Zhao, J. Yan, D. G. Mandrus, D. Xiao, W. Yao, and X. Xu, Nature Nanotechnol., doi: 10.1038/nnano.2013.151.

${ }^{24}$ Z. Y. Zhu, Y. C. Cheng, and U. Schwingenschlögl, Phys. Rev. B 84, 153402 (2011).

${ }^{25}$ X. Li, F. Zhang, and Q. Niu, Phys. Rev. Lett. 110, 066803 (2013).

${ }^{26}$ H.-Z. Lu, W. Yao, D. Xiao, and S.-Q. Shen, Phys. Rev. Lett. 110, 016806 (2013).

${ }^{27}$ F. Parhizgar, H. Rostami, and R. Asgari, Phys. Rev. B 87, 125401 (2013).

${ }^{28}$ Z. Gong, G.-B. Liu, H. Yu, D. Xiao, X. Cui, X. Xu, and W. Yao, Nat. Commun. 4, 2053 (2013).

${ }^{29}$ H. Rostami, A. G. Moghaddam, and R. Asgari, arXiv:1302.5901. ${ }^{30}$ F. Zahid, L. Liu, Y. Zhu, J. Wang, and H. Guo, arXiv:1304.0074.

${ }^{31}$ A. Kormanyos, V. Zolyomi, N. D. Drummond, P. Rakyta, G. Burkard, and V. I. Fal'ko, Phys. Rev. B 88, 045416 (2013).
${ }^{32}$ E. Cappelluti, R. Roldán, J. A. Silva-Guillén, P. Ordejón, and F. Guinea, arXiv:1304.4831.

${ }^{33}$ T. Cheiwchanchamnangij and W. R. L. Lambrecht, Phys. Rev. B 85, 205302 (2012).

${ }^{34}$ E. S. Kadantsev and P. Hawrylak, Solid State Commun. 152, 909 (2012).

${ }^{35}$ H. Zeng, B. Zhu, K. Liu, J. Fan, X. Cui, and Q. M. Zhang, Phys. Rev. B 86, 241301(R) (2012).

${ }^{36}$ K. Kośmider and J. Fernández-Rossier, Phys. Rev. B 87, 075451 (2013).

${ }^{37}$ Y. Song and H. Dery, Phys. Rev. Lett. 111, 026601 (2013).

${ }^{38}$ R. A. Bromley, R. B. Murray, and A. D. Yoffe, J. Phys. C 5, 759 (1972).

${ }^{39}$ L. F. Mattheiss, Phys. Rev. B 8, 3719 (1973).

${ }^{40}$ S. Lebègue and O. Eriksson, Phys. Rev. B 79, 115409 (2009).

${ }^{41}$ C. Ataca, H. Şahin, and S. Ciraci, J. Phys. Chem. C 116, 8983 (2012).

${ }^{42}$ Y. Ding, Y. Wang, J. Ni, L. Shi, S. Shi, and W. Tang, Phys. B (Amsterdam, Neth.) 406, 2254 (2011).

${ }^{43}$ C. Ataca and S. Ciraci, J. Phys. Chem. C 115, 13303 (2011).

${ }^{44}$ S. Bhattacharyya and A. K. Singh, Phys. Rev. B 86, 075454 (2012).

${ }^{45}$ J. Kang, S. Tongay, J. Zhou, J. Li, and J. Wu, Appl. Phys. Lett. 102, 012111 (2013)

${ }^{46}$ P. Johari and V. B. Shenoy, ACS Nano 6, 5449 (2012).

${ }^{47}$ H. Peelaers and C. G. Van de Walle, Phys. Rev. B 86, 241401(R) (2012).

${ }^{48}$ W. S. Yun, S. W. Han, S. C. Hong, I. G. Kim, and J. D. Lee, Phys. Rev. B 85, 033305 (2012).

${ }^{49}$ H. Shi, H. Pan, Y.-W. Zhang, and B. I. Yakobson, Phys. Rev. B 87, 155304 (2013).

${ }^{50}$ S. Horzum, H. Sahin, S. Cahangirov, P. Cudazzo, A. Rubio, T. Serin, and F. M. Peeters, Phys. Rev. B 87, 125415 (2013).

${ }^{51}$ W. Feng, Y. Yao, W. Zhu, J. Zhou, W. Yao, and D. Xiao, Phys. Rev. B 86, 165108 (2012).

${ }^{52}$ Y. Yao, L. Kleinman, A. H. MacDonald, J. Sinova, T. Jungwirth, D.-S. Wang, E. Wang, and Q. Niu, Phys. Rev. Lett. 92, 037204 (2004).

${ }^{53}$ D. Xiao, M.-C. Chang, and Q. Niu, Rev. Mod. Phys. 82, 1959 (2010).

${ }^{54}$ Y. Yao and Z. Fang, Phys. Rev. Lett. 95, 156601 (2005).

${ }^{55}$ H. Ochoa and R. Roldán, Phys. Rev. B 87, 245421 (2013).

${ }^{56}$ For the $\mathrm{WTe}_{2}$ monolayer, the band with the $d_{+1}$ orbital above the band gap is the fifth band above the band gap, whereas it is the fourth band above the band gap for the other $M X_{2}$ monolayers.

${ }^{57} \mathrm{R}$. Winkler, Spin-Orbit Coupling Effects in Two-Dimensional Electron and Hole Systems, Springer Tracts in Modern Physics, Vol. 191 (Springer, Berlin, 2003).

${ }^{58}$ D. Xiao, W. Zhu, Y. Ran, N. Nagaosa, and S. Okamoto, Nat. Commun. 2, 596 (2011).

${ }^{59}$ P.-O. Löwdin, J. Chem. Phys. 19, 1396 (1951).

${ }^{60}$ J. M. Luttinger and W. Kohn, Phys. Rev. 97, 869 (1955).

${ }^{61}$ G. Kresse and J. Furthmüller, Phys. Rev. B 54, 11169 (1996).

${ }^{62}$ G. Kresse and J. Furthmüller, Comput. Mater. Sci. 6, 15 (1996).

${ }^{63}$ P. E. Blöchl, Phys. Rev. B 50, 17953 (1994).

${ }^{64}$ G. Kresse and D. Joubert, Phys. Rev. B 59, 1758 (1999).

${ }^{65}$ J. P. Perdew, K. Burke, and M. Ernzerhof, Phys. Rev. Lett. 77, 3865 (1996).

${ }^{66}$ D. M. Ceperley and B. J. Alder, Phys. Rev. Lett. 45, 566 (1980).

${ }^{67}$ J. P. Perdew and A. Zunger, Phys. Rev. B 23, 5048 (1981). 